\title{
Lapurdum
}

LAPURDUM Euskal ikerketen aldizkaria | Revue d'études basques |

Revista de estudios vascos | Basque studies review

$5 \mid 2000$

Numéro V

\section{Note à propos du basque elika « aliment »}

\section{Michel Morvan}

\section{OpenEdition \\ Journals}

Édition électronique

URL : http://journals.openedition.org/lapurdum/1305

DOI : 10.4000/lapurdum.1305

ISSN : 1965-0655

Éditeur

IKER

\section{Édition imprimée}

Date de publication : 1 octobre 2000

Pagination : 181-182

ISBN : 2-84127-161-7

ISSN : $1273-3830$

\section{Référence électronique}

Michel Morvan, « Note à propos du basque elika « aliment »», Lapurdum [En ligne], 5 | 2000, mis en ligne le 01 juin 2009, consulté le 04 juillet 2020. URL : http://journals.openedition.org/lapurdum/1305 ; DOI : https://doi.org/10.4000/lapurdum. 1305 


\section{Note à propos du basque elika « aliment »}

Michel Morvan

UMR 5478

On pourra être surpris de constater qu'en basque le terme elika peut vouloir dire une chose et son contraire. En effet, si l'on suit les dictionnaires, on trouve pour cette base lexicale deux sens opposés « aliment » et « abstinence ». Azkue donne elikatu comme verbe ayant le sens de "nourrir, alimenter » et cite Garibay (Refranes, 1596, 57) : edo eguiaz nai guzurraz elikatuko naiz qu'il traduit " ou de vérité ou de mensonge je me nourrirai ». Suit une citation d'Axular (Gero, 3-22612) : doiaz elikatzen, iragaiten eta kontentatzen gara traduite par " nous nous alimentons, nous passons et nous nous contentons du nécessaire ».

Le deuxième sens conféré par Azkue à ce verbe est « s'abstenir » qu'il donne comme souletin avec la citation suivante : elika naite gauza horzaz « je puis m'abstenir de cela » (la citation est en fait d'Oihenart). Ce deuxième sens est complété par un troisième qui reprend l'idée d'alimentation : "s'alimenter légèrement » sans citation et enfin un quatrième sens est localisé en roncalais dans lequel apparaît la notion de "prendre ses dispositions 》 : elikatan dabila kura zerbait bergaintako, " celui-là a pris ses dispositions pour obtenir quelque chose ».

Les dérivés de la base *elika-, donnés par Azkue, ne modifient pas sensiblement les champs sémantiques couverts par celle-ci : elikatura " entretien, aliment », eliki « satisfaction », eliku-gabe " glouton».

Lhande cite elika "satisfait » et reprend avec elikatu les deux sens opposés « nourrir » et « s'abstenir ». Toutefois, un troisième sens indique « se passer de, goûter un peu ». Les dérivés sont les mêmes que ceux d'Azkue.

C'est en cherchant l'origine du terme basque elika que l'on va résoudre le problème posé par les deux sens opposés " nourrir, alimenter » et "s'abstenir ». On pourrait penser qu'il y a eu confusion de deux bases distinctes, avec influence possible de certains termes tels que eli, « troupeau de bétail » par exemple. Une telle influence est possible, mais par simple effet mécanique. En réalité le terme est un emprunt roman au castillan álica « aliment, bouillie ». Cela n'explique pas encore la notion d'abstinence. Pour cela il faut savoir que la bouillie est considérée comme un aliment pauvre, et plus particulièrement celle qui est faite à base de céréale. On comprend alors que le sens fondamental du terme basque est « aliment dont il faut 
se contenter » comme le laissaient entrevoir un des sens donnés par Azkue ( « s'alimenter légèrement ») et le troisième sens donné par Lhande (" se passer de, se contenter de, goûter un peu »). C'est cette dernière notion qui permet le passage sémantique de " se contenter de peu » à «s'abstenir ». Elle est corroborée par le fait que le terme castillan est lui-même issu directement du latin alica « bouillie d'épeautre ». Le terme est traduit par « semoule » dans le dictionnaire de Gaffiot qui cite Caton, De agricultura, 76, 1. Mais dans le Dictionnaire étymologique de la langue latine d'Ernout et Meillet, c'est bien « épeautre, bouillie ou boisson préparée avec ce grain » qui figure. Les auteurs ajoutent que ce terme s'est conservé en sarde, sicilien et espagnol (d'après Meyer-Lübke, Romanisches etymologisches Wörteburch, Heidelberg, 1935, 337).

Ernout et Meillet, après un détour par l'hypothèse d'un rapprochement avec le nom de la bière en indo-européen du nord, signalent le terme latin comme probable emprunt au grec alika, accusatif de alix, "gruau d'épeautre " (Athénée de Naukratis, Banquet des sophistes, 647). Ils ajoutent que le mot était, selon Pline (Naturalis historia, 22,128) d'usage récent en latin et aurait été introduit à Rome, avec la chose, par les médecins grecs si l'on en croit Hj. Frisk, Griechisches etymologisches Wörterbuch (Heidelberg, 1960-1972, s.u.) 